\title{
Gynecological disorders of adolescent girls at Kathmandu Medical College Teaching Hospital
}

\author{
Chanda Karki, Nira Singh Shrestha, Roshan T Rayamajhi \\ Kathmandu Medical College, Sinamangal
}

\begin{abstract}
A prospective OPD based study was done at Kathmandu Medical College Teaching Hospital (KMCTH) from $1^{\text {st }}$ September 2004 to $30^{\text {th }}$ August 2005.
\end{abstract}

Aim: To assess the gynecological disorders in adolescents and to study the menstrual disorders in the study group.

Result: A total of 1697 adolescent girls aged 13 to 19 years attending the gynecological outpatient department of KMCTH were included in the study. Their detail history was taken followed by general and systemic examinations and investigations like hemogram, coagulation profile, hormonal assays, and ultrasonography as per need were performed. Thus their gynecological problems including menstrual disorders were identified. With this information the details of their menstrual abnormalities were also analyzed.

Teenage pregnancy was the most frequently observed (56.33\%) diagnosis. Menstrual disorders were found to be the second most common (23.80\%) gynecological problem. They varied from amenorrhea (29.20\%) to menorrhagia. Dysfunctional uterine bleeding was the commonest etiology of menstrual dysfunction (204/286) in the study group.

Conclusion: Unsafe abortion and sexually transmitted infections were some of the other problems of adolescent girls. It is important to note that adolescents are not a homogenous group. Their needs vary with their sex, stage of development, life circumstances and the socioeconomic conditions of their environment. This is the time when they have the maximum burden of education and career building on their shoulders. They are the group who hesitate to seek services from traditional facilities. Therefore every health institution should initiate or strengthen adolescent friendly services and provide specialist care to this special group of people.

Key words: adolescence, gynecological problems, amenorrhea, menstrual dysfunction.

\section{Introduction}

WHO defines adolescents as individuals in the age group of 10-19 years $^{1}$. Gynecological problems of adolescents occupy a special space in the spectrum of gynecological disorders of all ages. This is because of the physical nature of the problems which are so unique, special, and specific for the age group, and also because of the associated psychological factors which are very important in the growth and psychological remodeling of someone in the transition between childhood and womanhood ${ }^{2}$.
Yet adolescent gynecology is a sub specialized area of gynecology which has still not been explored optimally. In this study, an attempt has been made to review the gynecological problems encountered in adolescents attending the gynecological outpatient department of Kathmandu Medical College Teaching Hospital and to analyze the menstrual abnormalities in this group.

\section{Methodology}

The total number of patients attending the gynecological outpatient department of KMCTH from 
$1^{\text {st }}$ September 2004 to $31^{\text {st }}$ August 2005 was recorded in the OPD register. Adolescents among them were identified. Out of them adolescents of only 13-19 years of age with gynecological problems were included in the study. Pregnancies of up to twenty eight weeks of gestation were also included as gynecological case. Detailed history of these adolescents with gynecological and other associated problems was taken. In addition to the general examination, height, weight, and secondary sex characteristics were recorded. Investigations such as hemogram, coagulation profile, hormonal assays (FSH, LH, prolactin, TFT), and pelvic ultrasound were done as and when indicated. Similarly the support of diagnostic laparoscopy was also taken in diagnosing few cases of primary amenorrhoea. With this information, gynecological problems and different types of menstrual disorders commonly found in them were analyzed. At the same time etiology of such menstrual abnormalities were also analyzed to meet the objectives of this study.

\section{Results}

It was found that a total of 13,691 women attended the gynecology out patient department (OPD) of KMCTH during the study period. Out of them, 1778 (12.98\%) were adolescents and 1697(12.39\%) of them were in the age group 13-19 years. Table 1 enumerates the various types of gynecological problems encountered in the study group. Teenage pregnancy was the most common $(56.33 \%)$ problem. Out of 956 pregnant teenagers one hundred and twenty seven (13.28\%) mothers came with unwanted pregnancy to seek comprehensive abortion care services. Menstrual disorders were the second most frequent cause for them seeking professional advice $(23.80 \%)$. Other conditions $(19.87 \%)$ noted were pelvic inflammatory disease (PID), ovarian cysts, leucorrhoea, abortion complications and sexual assault. Approximately three percent sought contraceptive advice.

Table I. Gynecological problems $(n=1697)$

\begin{tabular}{lcc}
\hline Types & Number & Percent $(\%)$ \\
\hline Teenage pregnancy & $956^{*}$ & 56.33 \\
Menstrual disorders & 404 & 23.80 \\
PID & 103 & 6.06 \\
Ovarian cysts/tumours & 65 & 3.83 \\
Leucorrhea & 62 & 3.66 \\
Abortion complications & 59 & 3.50 \\
Access to contraception & 45 & 2.66 \\
Sexual assault & 3 & 0.16 \\
\hline Total & 1697 & 100 \\
\hline
\end{tabular}

* CAC service seeker-127
Analysis of menstrual disorders in the study population are shown in Table 2 which reveals that menstrual disorders varied from amenorrhea in 118 (29.20\%) to menstrual dysfunction in 286(70.79\%). Ninety five girls had menorrhagia with seventeen of them having a hemoglobin of $<7 \mathrm{~g} / \mathrm{dl}$, twenty eight having $7-<9 \mathrm{~g} / \mathrm{dl}$ and 50 of them having $9-<11 \mathrm{~g} / \mathrm{dl}$.

Table 2. Menstrual disorders $(n=404)$

\begin{tabular}{lc}
\hline Menstrual disorders & Numbers \\
\hline Amenorrhea & \\
Primary & 31 \\
Secondary & 87 \\
\hline Total & $118(29.20 \%)$ \\
\hline
\end{tabular}

\section{Menstrual dysfunction}

Menorrhagia and metrorrhagia 95

Hypomenorrhea and oligomenorrhea 158

Dysmenorrhea 33

Total $286(70.79 \%)$

The etiological factors were analyzed in 118 cases of amenorrhea. Thirty one of them had primary amenorrhea while 87 had secondary amenorrhea, the causes of which are enlisted below.

Table 3. Etiology of amenorrhea $(n=\mid / 8)$

\begin{tabular}{cc}
\hline \multicolumn{1}{c}{ Etiology } & Number \\
\hline Primary amenorrhea & 6 \\
Mullerian agenesis & 6 \\
Imperforate hymen & 6 \\
Atresia upper third of vagina & 3 \\
Complete absence of vagina & 4 \\
Gonadal dysgenesis & 6 \\
Testicular feminizing syndrome & 31 \\
\hline Total
\end{tabular}

\section{Secondary amenorrhea}

Polycystic ovarian disease $\quad 16$

Post pill amenorrhea 6

Anaemia 13

Hypothyroidism 6

Marked psychological stress $\quad 13$

Microadenoma of pituitary gland 3

Tuberculosis 7

Granulosa cell tumor of the ovary 3

Diabetes 6

Premature ovarian failure 3

unexplained 11

\begin{tabular}{ll}
\hline Total & 87
\end{tabular}


Etiology of menstrual dysfunction are enlisted in Table 4. Dysfunctional uterine bleeding was the commonest (204/286) cause of menstrual dysfunction.

Table 4. Etiology of menstrual dysfunction $(n=286)$

\begin{tabular}{lc}
\hline \multicolumn{1}{c}{ Etiology } & Numbers \\
\hline Dysfunctional uterine bleeding & 204 \\
OCP users and breakthrough bleeding & 17 \\
Polycystic ovarian disease & 16 \\
Thyroid disorder & 6 \\
Idiopathic thrombocytopenic purpura & 3 \\
Marked psychological stress & 6 \\
Dysmenorrhoea & 34 \\
\hline Total & 286 \\
\hline
\end{tabular}

\section{Discussion}

This study shows that teenage pregnancy, menstrual disorders, PID, ovarian cysts, leucorrhea abortion related complications and sexual assault are some of the commonly encountered gynecological problems in adolescents. Some of them come for comprehensive abortion care and family planning services.

Teenage pregnancy is considered as high risk pregnancy. These girls often do not have safe sex and are vulnerable to sexually transmitted diseases. Moreover after getting pregnant, to avoid social problems, they go to quacks and undergo criminal abortion in unauthorized locations and land up with serious complications and chronic pelvic inflammatory disease which affect their reproductive health in future. Teenage pregnancies accounted for $56.33 \%$ in our study.

Menstrual disorders are the second most common gynecological problem $(23.80 \%)$ in adolescent girls. These range from amenorrhea to menorrhagia. Amenorrhea, both primary and secondary, was present in 118 girls. Mullerian agenesis was found in six out of thirty one girls with primary amenorrhea and one of them had solitary kidney. Six cases of primary amenorrhea were ultimately diagnosed as testicular feminizing syndrome through karyotyping. Three cases of vaginal agenesis presented with intermittent pain in abdomen and primary amenorrhea. They underwent vaginoplasty and are now having regular menstruation.

Most of the patients (16/87) suffering from secondary amenorrhea (duration 4-5 months) or oligomenorrhea were ultimately diagnosed to be cases of polycystic ovarian disease ( PCOD) based on clinical criteria of menstrual problems, features of hyperandrogenism, hormonal assay and sonography findings.
Premature ovarian failure as a cause of secondary amenorrhea is found in $10 \%$ cases below the age of $40^{3}$. It is rare in adolescents. In our study, three girls had secondary amenorrhea due to premature ovarian failure based on amenorrhea of more than 6 months, serum FSH of $43 \mathrm{IU} / \mathrm{L}$ - 45IU/L, and absence of follicles in the ovary on sonography.

DUB is not only restricted to the adult population but is more common in adolescents ${ }^{4}$. In as many as $95 \%$, abnormal vaginal bleeding is caused by DUB. It may take 2 to 5 years for the complete maturation of hypothalamic pituitary ovarian axis ${ }^{4}$. In the present study, out of the 286 girls suffering from menstrual problems, 204 were found to have DUB. Sixty-five cases were diagnosed as the cases of ovarian cyst and tumors, out of which 10 had dermoids and 54 had simple serous cyst adenoma. In one case, the tumor showed mixed solid and cystic areas on sonography. It turned out to be sertoli leydig cell tumor (arrhenoblastoma) on histopathology. The patient was given chemotherapy.

Three girls were brought to the hospital as victims of sexual assault and were referred to maternity hospital for medicolegal reasons. Fourteen other unmarried girls attending the OPD for other problems accepted being sexually active. These girls need to be counseled effectively so that they do not end up having unwanted pregnancy and criminal abortion.

\section{Conclusion}

A good number of adolescents are attending the Gynecology OPD at KMCTH. Most of them seek antenatal and safe abortion care services. Adolescents with various types of menstrual disorders are second in the list and they also need to be taken care of on a priority basis. Careful and detailed history, thorough examination and a very good pathology lab support are required to diagnose the menstrual disorders accurately and to identify their causes. Although psychosocial counseling is often sought by adolescents in developed countries our data does not include any case solely seeking psychosocial support. However, we have endeavored to provide relevant counseling to every adolescent who visited us for whatever primary reason. Awareness of the availability of adolescent friendly services where they can seek specialist psychosocial support would likely result in adolescents coming in for these services as well.

Adolescent gynecology is not a new subject. But it needs increasing awareness and further attention. Since the problems are specific to this group, setting up of separate adolescent clinics is desirable for efficient management. 


\section{Acknowledgement}

We are thankful to all the adolescents attending the Gynecology outpatient department of Kathmandu Medical College during the study period. Without them this analysis would not have been possible.

We are extremely thankful to our Head of the department Prof. S M Padhye who has not only permitted to publish this paper but also have been a great source of inspiration for carrying out various academic activities in the department.

We would also like to convey our sincere thanks to all the faculty members, lecturers, registrars, house officers and interns from the department of Obstetrics and Gynecology who has supported this study.

\section{References}

1. National adolescent Health and Development strategy developed by Family Health Division, Department of Health Services, Ministry of Health, His Majesty's Government Nepal in 2000.

2. Goswami S, Dutta R, Sengupta S. Aprofile of adolescent girls with gynaecological problems. The journal of obstetrics and Gynaecology of India. July August 2005; vol 55, no 4:353-355

3. Coulam CB, Adamson SC, Annegers JF. Incidence of premature ovarian failure obstet Gynaecol 1986,67:604-606

4. Falcone T, Desjardins C, Bourque $\mathrm{J}$ et al. Dysfunctional uterine bleeding in adolescents $\mathrm{J}$ Reproductive Med 1994;39:7761:4 